\title{
Altered Expression of Angiopoietins During Blood-Brain Barrier Breakdown and Angiogenesis
}

\author{
Nima Nourhaghighi, Krystyna Teichert-Kuliszewska, Jaime Davis, \\ Duncan J. Stewart, and Sukriti Nag
}

Toronto Western Research Institute, University Health Network (NN, JD, SN), and Terrence Donnelly Heart Center, St. Michael's Hospital (KT-K, DJS), and Departments of Laboratory Medicine and Pathobiology and Medicine (KT-K, DJS), University of Toronto, Toronto, Canada

\begin{abstract}
SUMMARY: Angiopoietin-1 (Ang-1) and angiopoietin-2 (Ang-2) belong to a novel family of endothelial growth factors that function as ligands for the endothelial-specific receptor tyrosine kinase, Tie-2. Ang-1 reduces endothelial permeability of noncerebral vessels and has a major role in vascular stabilization and maturation, whereas Ang-2 is thought to be an endogenous antagonist of the action of Ang-1 at Tie-2. Expression of these ligands at the mRNA and protein level were studied during both blood-brain barrier (BBB) breakdown and cerebral angiogenesis occurring in the rat cortical cold-injury model by RT-PCR analysis and immunohistochemistry respectively, during a time course of 6 hours to 6 days. In addition, immunohistochemical detection of fibronectin was used to detect BBB breakdown the lesion site and dual labeling was used to determine whether the vessels demonstrating BBB breakdown expressed endothelial Ang-1 or Ang-2. Endothelial Ang-1 and Tie-2 proteins were present in all cerebral vessels of normal brain including those of the choroid plexuses, whereas both these proteins as well as Ang-2 were present in choroid plexus epithelium and in ependymal cells, suggesting that angiopoietins have an autocrine effect on these cell types as well. In contrast, in the early phase after injury during the known period of BBB breakdown, increased Ang-2 mRNA and protein and decreased endothelial Ang-1 and Tie-2 proteins were observed. Two to 6 days after injury, the progressive increase in Ang-1 mRNA and protein and the decrease in Ang-2 coincided with cerebrovascular angiogenesis. Confocal microscopy showed colocalization of both Ang-1 and Ang-2 in endothelium of lesion vessels, and our observation of colocalization of Ang-1 and Ang-2 in polymorphonuclear leukocytes and macrophages has not been reported previously. This study demonstrates that Ang-1 is an important factor in maintaining normal homeostasis in the brain. Thus Ang-1 therapy may have therapeutic potential in reducing BBB breakdown and the ensuing edema after massive brain injury. (Lab Invest 2003, 83:1211-1222).
\end{abstract}

A ngiopoietins are a family of glycoproteins that play a major role in the development and integrity of blood vessels (Davis et al, 1996; Maisonpierre et al, 1997; Suri et al, 1996). Angiopoietin-1 (Ang-1) is a 498-amino acid glycoprotein, the gene for which has been localized to chromosome 8q22.3-q23 (Cheung et al, 1998; Grosios et al, 1999). Angiopoietin-2 (Ang-2) is a 496-amino acid glycoprotein with $60 \%$ homology with Ang-1, and its gene is located on chromosome 8p23.1 (Cheung et al, 1998; Grosios et al, 1999). Although both Ang-1 and Ang-2 bind to the Tie-2 receptor, which is ubiquitously expressed in vascular endothelium (Wong et al, 1997), they mediate different effects on binding to this receptor. Binding of Ang-1 to Tie-2 causes its phosphorylation and promotes vascular maturation and quiescence by inducing association of endothelium with periendothelial

\section{DOI: 10.1097/01.LAB.0000082383.40635.FE}

Received April 9, 2003.

Supported by Heart and Stroke Foundation of Ontario Grant NA 4437 and an Ontario Neurotrauma Foundation Scholarship to Nima Nourhaghighi.

Address reprint requests to: Dr. S. Nag, EW5-510, Neuropathology, Toronto Western Hospital, 399 Bathurst Street, Toronto, Ontario M5T 2S8, Canada.E-mail:nag@uhnres.utoronto.ca supporting cells (Audero et al, 2001; Davis et al, 1996; Suri et al, 1996). Ang-2 does not phosphorylate Tie-2 but is a putative natural antagonist of Ang-1, causing vascular remodeling by counteracting the blood vessel maturation/stabilization mediated by Ang-1 (Maisonpierre et al, 1997). Ang-1 and Ang-2 have other reciprocal effects. As an example, Ang-1 has an antiapoptotic effect on endothelial cells through the Akt/survivin/PI-3' kinase pathway (Hayes et al, 1999; Kwak et al, 1999; Papapetropoulos et al, 2000), whereas Ang-2 is thought to promote apoptosis because it is detected just before the onset of apoptosis in vascular cells (Cohen et al, 2001; Zagzag et al, 2000).

Ang-1 and Tie-2 knockouts display defects in remodeling and integrity of the primitive vascular plexus, leading to embryonic lethality (Patan, 1998; Suri et al, 1996). Transgenic overexpression of Ang-1 induces leakage resistance in blood vessels (Thurston, 2002; Thurston et al, 1999) and increased vascularization (Suri et al, 1998). The phenotype of transgenic Ang-2 overexpression is similar to the Ang-1 and Tie-2 knockouts, involving severe disruptions in vascular development (Maisonpierre et al, 1997). Thus, angiopoietin signaling seems to be indispensable for normal vascular development and homeostasis. 
Brain injury is associated with both blood-brain barrier (BBB) breakdown and angiogenesis (Nag, 2002). It is conceivable that Ang-1 maintains BBB integrity, because it induces a leakage-resistance phenotype in peripheral vessels (Thurston et al, 1999), whereas Ang-2 may be involved in BBB breakdown and cerebrovascular angiogenesis after injury, because it has been implicated in peripheral vascular remodeling and angiogenesis (Cohen et al, 2001; Goede et al, 1998). Although Ang-1 mRNA has been localized in normal human (Hashimoto et al, 2001) and rat (Mandriota et al, 2000) brain and up-regulation of angiopoietins are known to occur in neoplastic cerebral angiogenesis (Audero et al, 2001; Stratmann et al, 1998; Zagzag et al, 1999), little is known about their role during BBB breakdown and nonneoplastic cerebral angiogenesis.

This study characterizes Ang-1, Ang-2, and Tie-2 mRNA and protein expression in cerebral vessels of normal rat brain and in the rat cortical cold-injury model during a period of 6 hours to 6 days. Our previous studies have established the time course of BBB breakdown and angiogenesis in the rat cortical cold-injury model, making it a suitable model for studies of angiopoietin expression during both these processes (Nag, 1996; Nag et al, 2001, 2002). Ang-1, Ang-2, and Tie-2 mRNA were detected by RT-PCR, whereas their proteins were localized using immunohistochemistry. Immunohistochemical detection of fibronectin was used as a marker of BBB breakdown, and dual labeling was used to detect whether vessels demonstrating BBB breakdown to protein showed endothelial Ang-1 or Ang-2 protein. Colocalization of Ang-1 and Ang-2 in brain was also studied by dual labeling for both these proteins.

\section{Results}

\section{Morphology of the Cold Lesion}

The morphology of the cortical cold lesion at the different time points was similar to our previous findings (Nag, 1996; Nag et al, 2001, 2002). At 6 hours, a central area of coagulative necrosis extended from the pial surface into the fourth cortical layer at the lesion site. Neurons in this area showed nuclear pyknosis and shrunken cytoplasm and neuronal loss was present in all rats killed at Day 2. The onset of angiogenesis was marked by an increase in the number of endothelial cells around preexisting vessels. At Day 4, profiles of neovessels were interspersed among the endothelial cells. At Day 6, neovessels were present throughout the lesion. The time course of the inflammatory response was consistent with an initial polymorphonuclear leukocyte (PMN) response being detected as early as 6 hours followed by a macrophage response, which was prominent along the margins of the lesion and peaked at Day 4.

\section{Expression of Ang-1, Ang-2, and Tie-2 mRNA}

Baseline Ang-1, Ang-2, and Tie-2 expression was detected in the brain cortex of control rats (Fig. 1, A to
C). At 6 hours, Ang-1 mRNA was not significantly altered as compared with values of control rats, whereas Ang-2 mRNA increased by $7.0 \pm 2.4$-fold $(p$ $<0.005$ ) (Fig. 1, B and C). Thereafter, Ang-1 mRNA in the lesion area showed a progressive increase, whereas Ang-2 mRNA displayed a progressive decrease. At Day 6, Ang-1 mRNA expression increased to $3.5 \pm 0.8$-fold $(p<0.001)$ relative to control levels, whereas Ang-2 mRNA expression was similar to that of control rats (Fig. 1, A to C). Tie-2 mRNA expression remained constant throughout the time course (Fig. 1A).

\section{Localization of Ang-1, Ang-2, and Tie-2 Proteins}

Control Rats. Marked endothelial Ang-1 immunostaining was observed in all brain vessels (Fig. 2A), whereas only mild Ang-2 immunoreactivity was present in endothelium and perivascular cells of occasional vessels. Areas with vessels having no BBB characteristics such as the choroid plexuses, median eminence, and area postrema, also showed vessels with endothelial Ang-1 but not Ang-2 immunoreactivity (Fig. 2B). Choroid plexus epithelium, however, showed granular immunostaining for both Ang-1 and Ang-2, and both these proteins were also localized in the luminal plasma membrane and cilia of the ependymal cells lining the third and fourth ventricles (Fig. 2, B and $\mathrm{C}$ ). The cilia of ependyma lining the roof of the fourth ventricle showed particularly high Ang-2 immunoreactivity. Both antibodies used to obtain Ang-2 immunostaining gave similar results.

Endothelial Tie-2 protein was present in all intracerebral vessels including the vessels of the choroid plexuses. In addition Tie-2 immunoreactivity was also present in the luminal plasma membrane of choroid plexus epithelium and ependymal cells including the cilia of the latter cells.

Cold-Injured Rats. Cerebral vessels: At 6 hours, endothelial Ang-1 and Tie-2 immunostaining was decreased in lesion vessels (Fig. 2D). At Day 2, there was moderate endothelial Ang-1 immunoreactivity in lesion and peri-lesion vessels, whereas endothelial Ang-2 immunostaining was still only mildly detectable. At Day 3, moderate endothelial Ang-1 immunoreactivity persisted in lesion vessels, whereas endothelial Ang-2 immunoreactivity was significantly more prevalent compared with Day 2 after injury (Fig. 2, E and F). During the peak of vascular remodeling at Day 4, lesion and overlying pial vessels showed marked endothelial Ang-1 and Ang-2 immunoreactivity (Fig. 2, $\mathrm{G}$ and $\mathrm{H}$ ). During the later remodeling phase at Day 6, most of the neovessels showed endothelial Ang-1 and only occasional vessels showed endothelial Ang-2 (Fig. 2, $\mathrm{J}$ and $\mathrm{K}$ ).

Quantitative morphometry was performed to determine the number of vessels showing Ang-1 and Ang-2 immunoreactivity at Days 2 and 4. At Day 2, the number of lesion vessels showing endothelial Ang-1 immunoreactivity was significantly decreased (52 \pm $2 \% ; p<0.001)$ as compared with the vessels of control rats $(99 \pm 0.2 \%)$, of which showed endothelial Ang-1 (Fig. 3). At Day 4, the percentage of Ang-1 
A

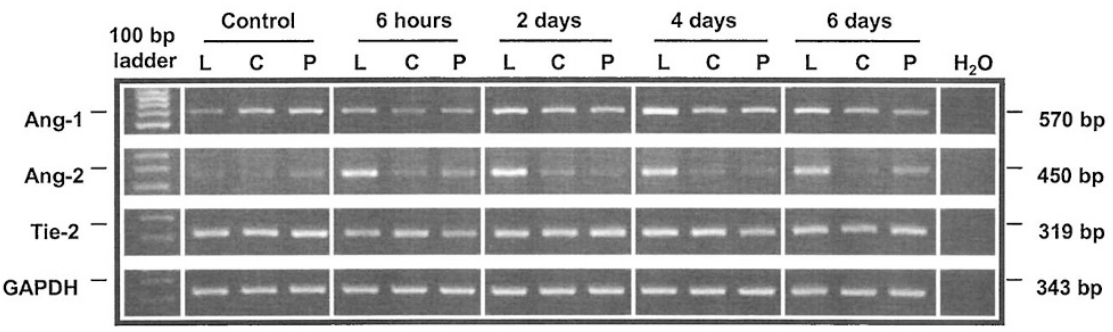

B

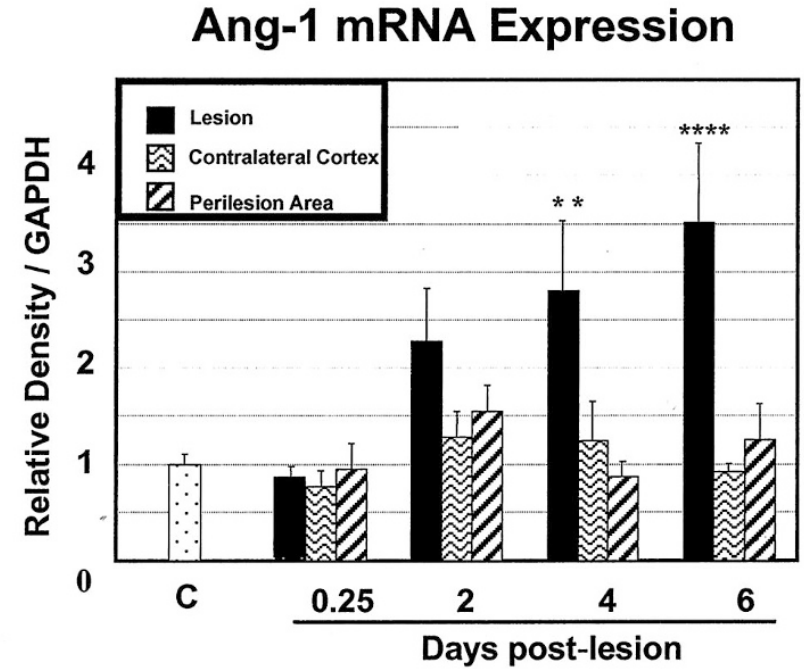

C

\section{Ang-2 mRNA Expression}

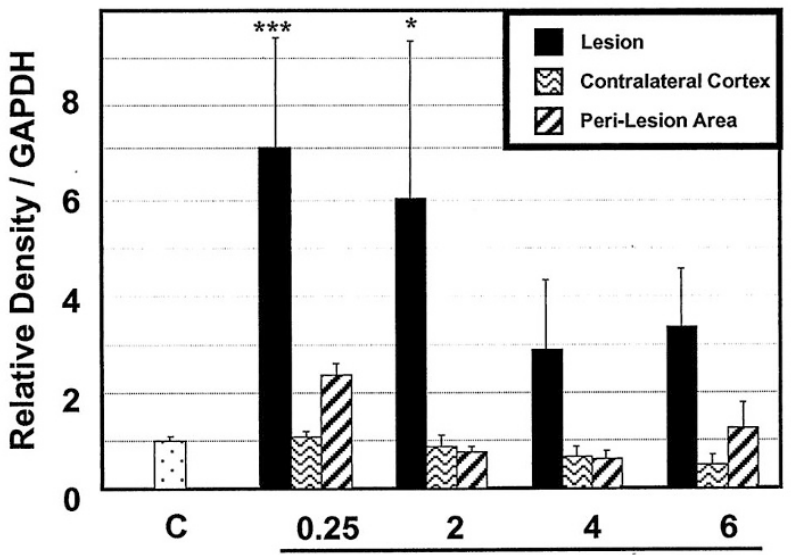

Days post- lesion

Figure 1.

A representative RT-PCR analysis shows angiopoietin-1 (Ang-1) (570 bp), angiopoietin-2 (Ang-2) (450 bp), Tie-2 (319 bp), and glyceraldehyde-3-phosphate dehydrogenase (343 bp) mRNA expression in the cortex of control and cold-injured rats at 6 hours and at 2, 4, and 6 days after injury (A). For each rat, samples were taken from the lesion area $(L)$, the corresponding area of the contralateral cortex $(C)$, and the peri-lesion area $(P)$. Basal expression of Ang-1, Ang-2, and Tie-2 is observed in the cerebral cortex of control rats. Progressive increase in Ang-1 mRNA signal intensity is observed during the 6-day period of observation, compared with control levels. The Ang-2 mRNA signal intensity is increased as early as 6 hours after injury compared with control levels, and then a progressive decrease is observed. Graphical representations of Ang-1 (B) and Ang-2 (C) mRNA after cortical cold injury are shown. Ang-1 mRNA is not significantly different from contro values at 6 hours and Day 2 after injury. However, at Days 4 and 6 , Ang- $1 \mathrm{mRNA}$ is increased $2.8 \pm 0.7$-fold $(p<0.01)$ and $3.5 \pm 0.8$-fold $(p<0.001)$, respectively, compared with control brains. Ang-2 mRNA shows a $7.0 \pm 2.4$-fold up-regulation as early as 6 hours after injury $(p<0.005)$ and a $6.1 \pm 3.3$-fold increase at Day 2 after injury. At Days 4 and 6 after injury, Ang-2 mRNA levels are not significantly different from control levels. A significant inverse relationship between Ang-1 and Ang-2 mRNA levels during 6 hours to 6 days after injury is implied by their Pearson's correlation coefficient of -0.871 . Statistical significance is denoted by asterisks: ${ }^{\star} p<0.05,{ }^{* \star} p<0.01,{ }^{* \star \star} p<0.005,{ }^{* \star \star *} p<0.001 . n=20$ for control rats and $n=5$ for each time point. Bars represent mean relative normalized optical density multiplied by area \pm SE. 

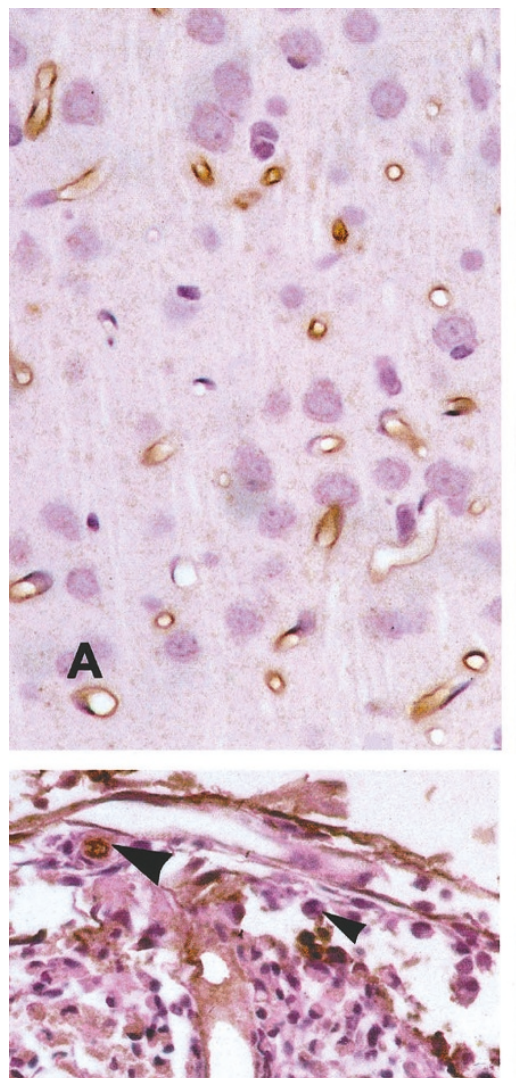

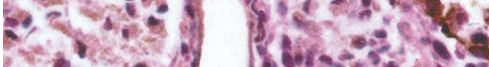

D.

$20^{2}$ of

provert biof an?

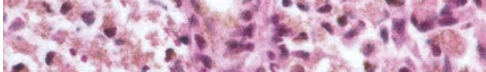

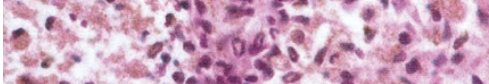

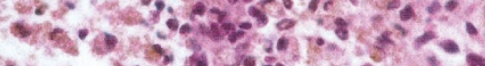

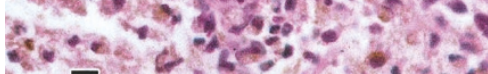

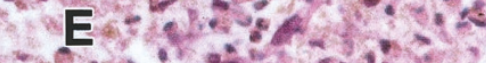

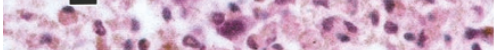

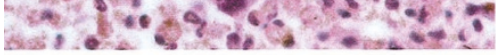

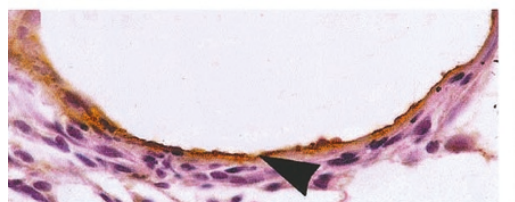

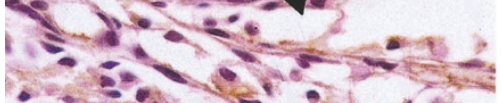

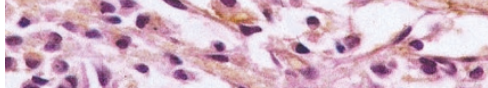

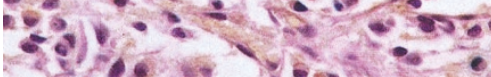

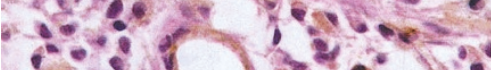

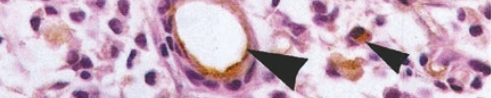
- 85 c5: ${ }^{-10}$ $-1007000$. co:0 B.

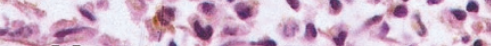

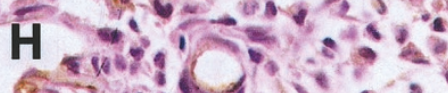
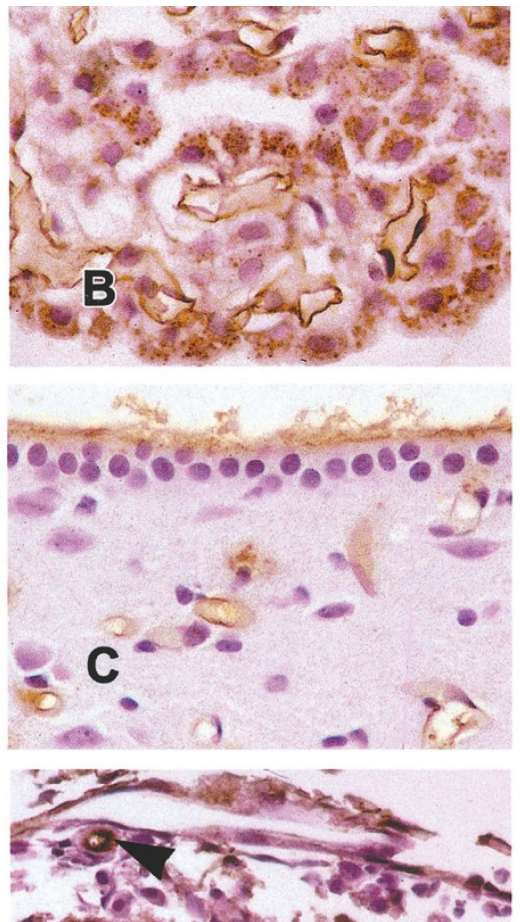

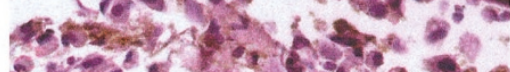

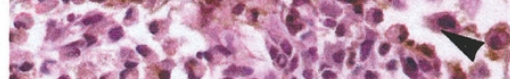

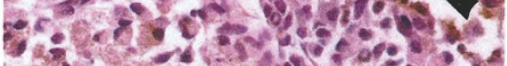

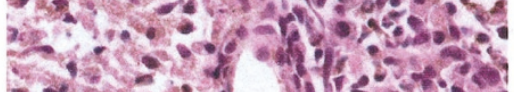

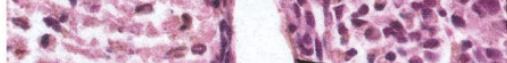

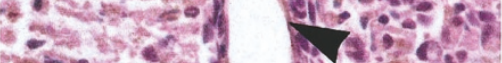

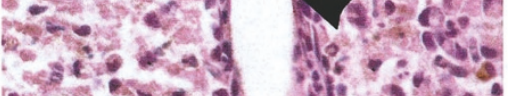
2.

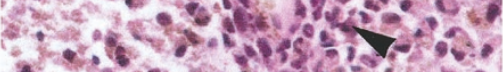

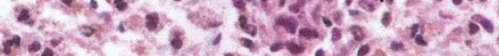

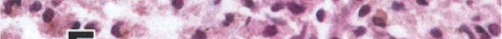

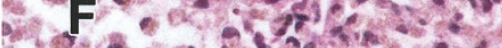

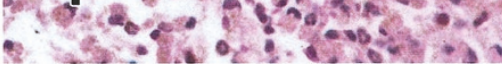

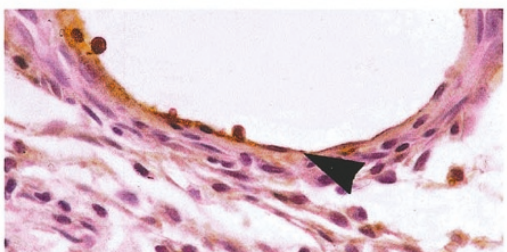
(5.) $\sin ^{2}-x^{2}$

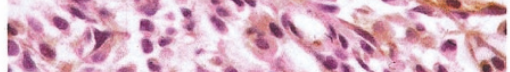

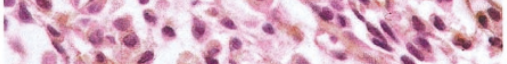

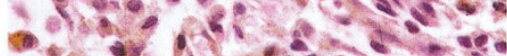

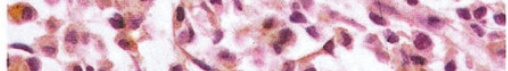

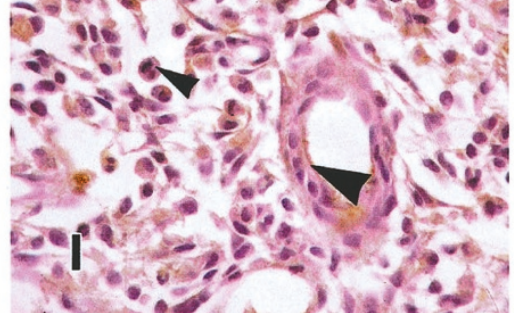
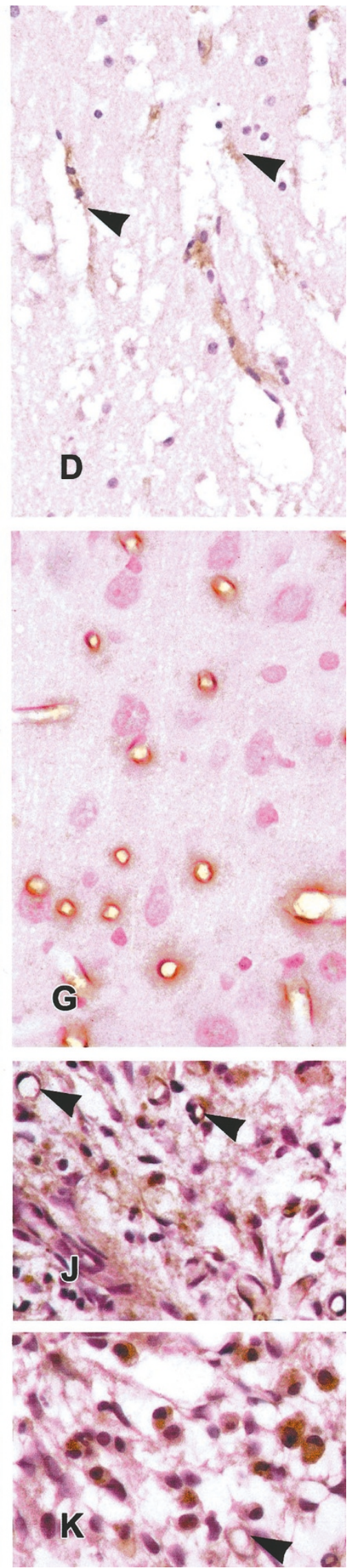
immunoreactive vessels increased to $87 \pm 5 \%$, which is not significantly different from control levels but is significantly increased compared with the number of positive vessels at Day 2 after injury $(p<0.001)$. Endothelial Ang-2 immunoreactivity at Day 2 was observed in $19 \pm 3 \%$ of lesion vessels, which is a significant increase compared with immunoreactivity in $0.5 \pm 0.2 \%$ of vessels of control rats $(p<0.01)$. At Day 4 after injury, endothelial Ang-2 immunoreactivity increased to $89 \pm 4 \%$ of lesion vessels; this is significantly higher $(p<0.001)$ compared with vessels of control rats and those of rats killed at Day 2 after injury (Fig. 3).

Endothelial Tie-2 immunostaining in lesion vessels at the margins of the necrotic area did not differ from those in the surrounding cortex (Fig. 2G). At 6 hours, vessels in the central necrotic area failed to show Tie-2 immunostaining. There was a progressive increase in the number of neovessels showing endothelial Tie-2 protein from Day 3 onward. At Day 6, Tie-2 immunoreactivity was present in all neovessels.

Inflammatory cells: Polymorphonuclear leukocytes infiltrating into the lesion area at 6 hours showed cytoplasmic Ang-1 and Ang-2 immunoreactivity. At Day 2, $78 \pm 1 \%$ of PMNs showed marked cytoplasmic Ang-1 immunoreactivity, whereas $84 \pm 1 \%$ showed marked Ang-2 immunoreactivity. At this time period, fewer macrophages were present and $46 \pm 10 \%$ of macrophages showed cytoplasmic Ang-1, whereas $30 \pm 3 \%$ showed cytoplasmic Ang-2. At Days 3 and 4, cytoplasmic immunoreactivity in PMNs and macrophages was marked (Fig. 2, $\mathrm{H}$ and I). At Day 4, $77 \pm$ $10 \%$ of PMNs showed Ang-1 immunoreactivity and almost the same number showed Ang-2 (76 $\pm 10 \%)$. The number of macrophages was increased as compared with Day 2, and $76 \pm 7 \%$ of macrophages showed cytoplasmic Ang-1, whereas $83.0 \pm 5 \%$ showed Ang-2 immunoreactivity. At Day 6, Ang-1 and Ang-2 immunoreactivity in macrophages was similar to Day 4 (Fig. 2K).

\section{Colocalization of Ang-1 and Ang-2}

Confocal microscopy showed a strong signal for Ang-1 in endothelium of vessels of control rats, which overshadowed the weak signal produced by Ang-2 protein (Fig. 4, A and B). Normal choroid plexus vessels also showed a strong Ang-1 signal, whereas choroid plexus epithelium showed colocalization of Ang-1 and Ang-2 (Fig. 4C).

Inflammatory cells within the lesion including both PMNs and macrophages showed cytoplasmic colo-

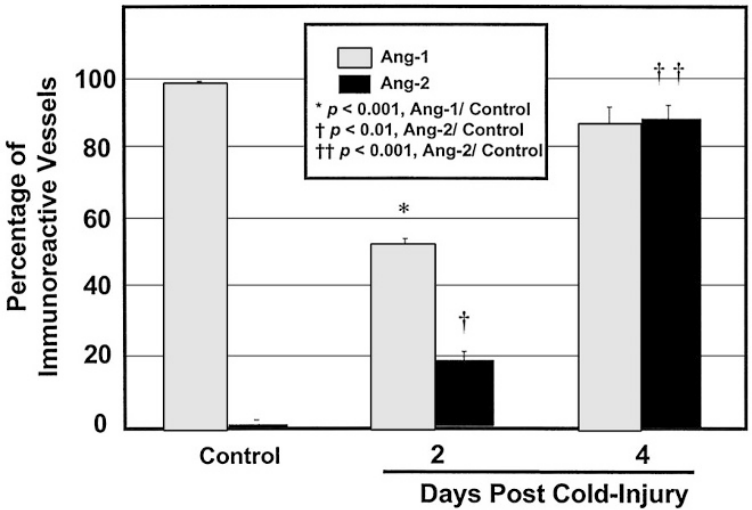

Figure 3

Graph showing the number of vessels with endothelial Ang-1 and Ang-2 immunoreactivity in cortex of control rats and in the cold-injury site at Days 2 and 4. Most of the vessels in the cortex of control rats showed endothelial Ang-1 (99 $\pm 0.2 \%)$, whereas endothelial Ang-2 is present in only occasional vessels $(0.5 \pm 0.2 \%)$. At Day 2 , the number of vessels showing endothelial Ang-1 has decreased to $52 \pm 2 \%\left({ }^{*} p<0.001\right)$, whereas those showing Ang-2 have increased to $19 \pm 3 \%(\dagger p<0.01)$. At Day 4 , vessels showing endothelial Ang-1 have increased to $88 \pm 5 \%$, whereas those showing Ang-2 are still significantly elevated ( $89 \pm 4 \%$ ), as compared with Ang-2 in normal cortex $(\dagger+p<0.001)$. The bars represent mean percentage of immunoreactive vessels in three high-power fields $\left(0.233 \mathrm{~mm}^{2}\right) . n=5$ in each group.

calization of Ang-1 and Ang-2 proteins (Fig. 4, D to F). Astrocytes in the immediate peri-lesional area also showed colocalization of Ang-1 and Ang-2 (Fig. 4G). Serial sections showed colocalization of Ang-1 and Ang-2 in the same pial and intracerebral vessels at Days 3 and 4 (Fig. 2, $\mathrm{E}$ to $\mathrm{H}$ ). This was further supported by confocal microscopy, which demonstrated colocalization of both Ang-1 and Ang-2 in endothelium of lesion vessels at Day 4 (Fig. 4H).

\section{BBB Breakdown and Ang Localization}

BBB breakdown at the lesion site was detected by immunostaining for fibronectin. Control rats showed fibronectin immunoreactivity in the serum proteins present in the lumina of occasional poorly perfused vessels, in the adventitia of pial vessels, and in the pia-arachnoid membrane. The time course of BBB breakdown in cold-injured rats was similar to our previous observations (Nag, 1996, 2002; Nag et al, 2002). An immediate phase of BBB breakdown of lesion vessels occurred, and at 6 hours diffuse fibronectin immunoreactivity was present at the lesion site and in continuity in the underlying white matter and that of the contralateral hemisphere. A more delayed phase of BBB breakdown involved both le-

\section{Figure 2.}

Endothelial Ang-1 immunoreactivity is present in all vessels of the cerebral cortex (A) and in choroid plexus vessels (B). B, Punctate Ang-1 immunoreactivity is also present in choroid plexus epithelial cells. C, The luminal plasma membrane of ependymal cells and their cilia show Ang-1 immunoreactivity, which is also present in endothelium of periventricular vessels. D, Cold-injury site showing decreased endothelial Ang- 1 immunoreactivity (arrowheads) at 6 hours after injury. E and F, At Day 3, serial sections show colocalization of Ang-1 (E) and Ang-2 (F) in the endothelium of intracerebral vessels (large arrowheads). Macrophages show cytoplasmic immunoreactivity for both Ang-1 and Ang-2 (small arrowheads). Dystrophic calcification is present along the left hand border of the photomicrograph, which accounts for the nonspecific brown staining between the inflammatory cells. G, No alteration was observed in the endothelial Tie-2 immunoreactivity in the peri-lesional area of cold-injured rats as compared with control rats. $\mathrm{H}$ and I, Colocalization of Ang-1 (H) and Ang-2 (I) in endothelium of pial and intracerebral arterioles was also present at Day 4 (large arrowheads). Note persistence of cytoplasmic Ang-1 and Ang-2 immunoreactivity in macrophages (small arrowheads). At Day 6, most of the neovessels showed endothelial Ang-1 (J, arrowheads) and few showed mild Ang-2 (K, arrowhead) immunoreactivity. Marked Ang-2 immunoreactivity was still evident in the macrophages present. $A$ to $\mathrm{K}, \times 600$. 

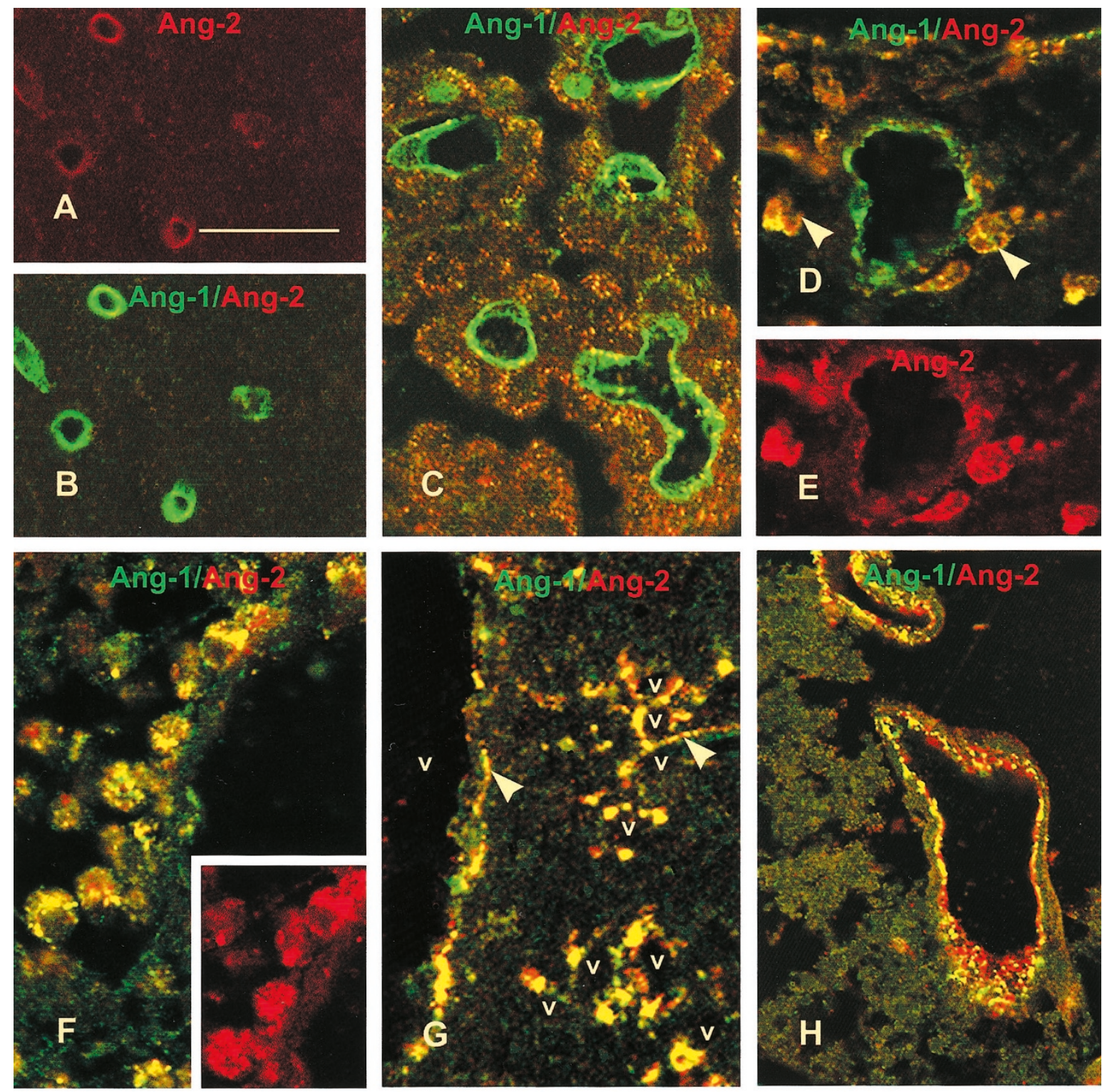

\section{E}
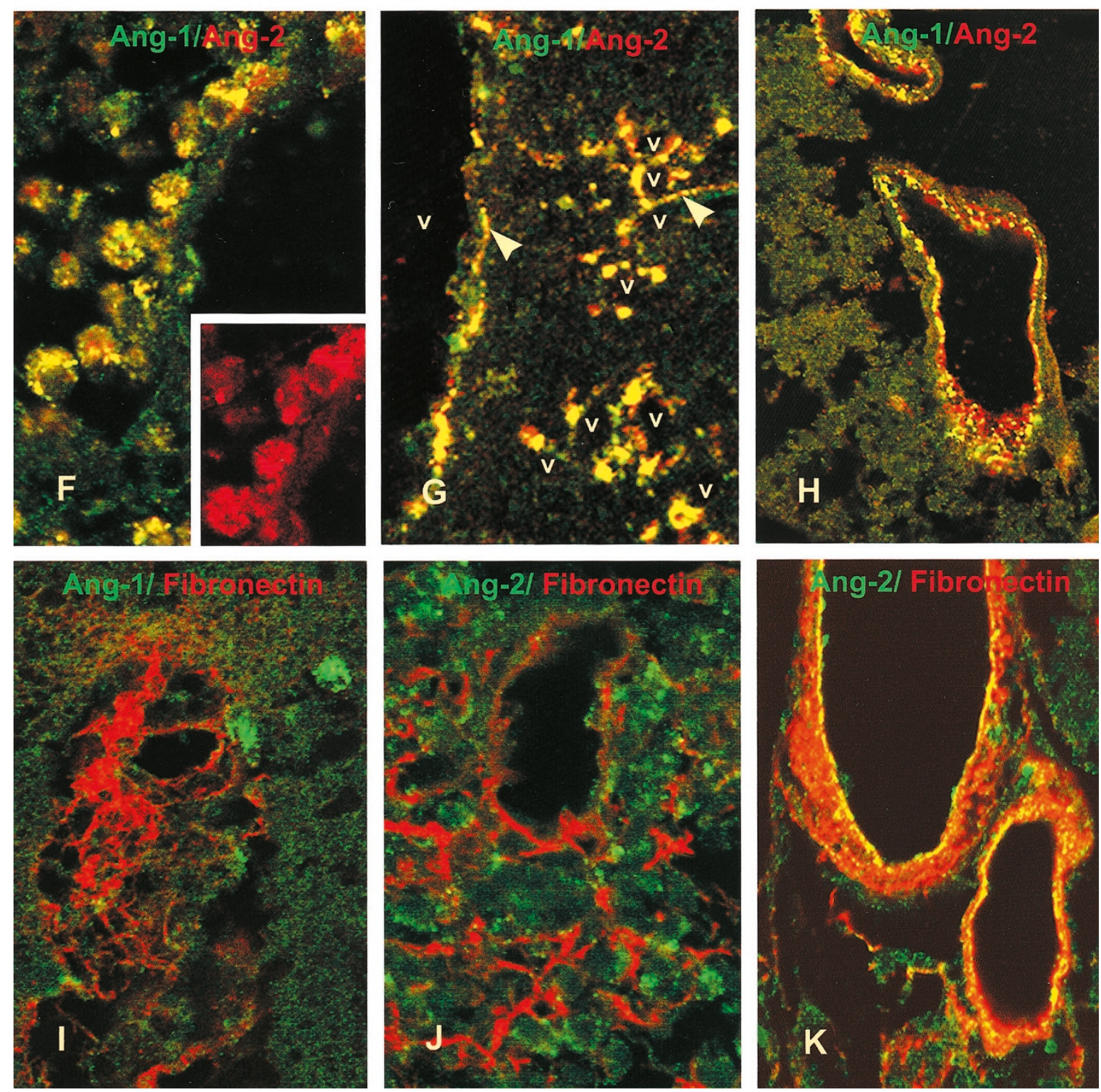
sion and peri-lesion vessels, and this was prominent between Days 2 and 4 (Fig. 4I). At Day 4, protein extravasation was localized to the lesion site and marked fibronectin staining was present at the periphery of the newly formed endothelial cells at the lesion site (Fig. 4J). Fibronectin immunoreactivity showed a progressive decline and at Day 6, four of five rats showed protein localizations that were similar to controls, whereas one rat showed mild fibronectin immunoreactivity in a focal area at the margin of the lesion and normal brain.

Dual staining for Ang-1 or Ang-2 and fibronectin showed that vessels with BBB breakdown at Days 2 and 4 failed to show endothelial Ang-1 or Ang-2 (Fig. 4, I and J). Nonpermeable pial and intracerebral lesion vessels did show endothelial Ang-2 at Days 2 and 4 (Fig. 4K).

\section{Discussion}

This study demonstrates endothelial Ang-1 and Tie-2 proteins in all cerebral vessels of normal brain including those of the choroid plexuses, whereas both these proteins and Ang-2 were present in choroid plexus epithelium and in ependymal cells. In contrast, in the early phase after injury during the known period of BBB breakdown, increased Ang-2 mRNA and protein and decreased endothelial Ang-1 and Tie-2 proteins were observed. Two to 6 days after injury, the progressive increase in Ang-1 mRNA and protein and the decrease in Ang-2 coincided with cerebrovascular angiogenesis.

\section{Ang-1, Ang-2, and Tie-2 mRNA Expression in Normal Brain}

Basal Ang-1 and Ang-2 mRNA expression in normal rat cortex was detected using RT-PCR, with the Ang-1 signal being more prominent than the Ang-2 signal. Because both reactions were performed for the same number of cycles, and assuming that the sensitivities of the probes for their targets are similar, Ang-1 mRNA expression exceeded Ang-2 mRNA expression in normal brain. Similar findings were observed in normal brain vessels by other authors using RT-PCR (Hashimoto et al, 2001; Mandriota et al, 2000). Studies using in situ hybridization provide conflicting data about Ang-1 mRNA localization in the brain. Ang-1 and Ang-2 mRNA were localized in neurons, whereas
Ang-1 was also observed in vessels (Stratmann et al, 1998). Subsequent studies reported only Ang-1 mRNA in glia and cerebellar Purkinje cells (Beck et al, 2000; Hashimoto et al, 2001) but not in blood vessels, whereas another study failed to demonstrate Ang-1 or Ang-2 mRNA in normal brain (Zagzag et al, 1999). These inconsistent results may be a result of technical difficulties such as fixation techniques and probe sensitivity. In this study, RT-PCR was chosen instead of in situ hybridization because the former is associated with less technical variability.

Cellular localization of Ang-1 and Ang-2 proteins in normal rat brain performed by immunohistochemistry showed marked endothelial Ang-1 immunoreactivity in brain vessels, whereas only mild Ang-2 immunoreactivity was detectable in occasional vessels. These Ang-1 findings differ from a recent study of human brain, which shows Ang-1 protein in few neurons and only mild immunoreactivity in vessels of controls (Audero et al, 2001). This is probably a result of the fact that the antibody dilution used in this study was twice that used in the present study because tumors, which were being studied, have a much higher signal for angiopoietins than normal brain.

The presence of angiopoietins in normal cerebral vessels has important implications for cerebral homeostasis. Ang-1 is known to increase expression of endothelial junctional proteins and alter their phosphorylation in in vitro studies of noncerebral endothelium (Gamble et al, 2000) and to promote association of the vascular endothelium with perivascular cells in the peripheral vasculature (Audero et al, 2001; Patan, 1998; Suri et al, 1996). Therefore, constitutive Ang-1 expression in normal cerebral endothelium may maintain endothelial tight junctions, thus maintaining BBB integrity. However, Ang-1 is also present in vessels of certain brain regions where vessels do not have BBB characteristics, such as the area postrema and the choroid plexuses. In addition, granular Ang-1 and Ang-2 immunoreactivity was also observed in the choroid plexus epithelium, whereas the Tie-2 receptor was localized to the luminal plasma membrane of epithelium. Ang-1, Ang-2, and Tie-2 proteins were also localized at the luminal plasma membrane and adjacent cilia of ependymal cells. Choroid plexus epithelium and ependymal cells are involved in production of cerebrospinal fluid and fluid permeability, respectively; hence it is possible that angiopoietins

\section{Figure 4.}

Confocal image of cortex of a control rat showing mild endothelial Ang-2 signal (A), which is overshadowed by the strong Ang-1 signal when the red and green channels are merged (B). C, Merged confocal image showing strong endothelial Ang-1 signal in choroid plexus vessels. The choroidal epithelial cells show yellow punctate immunoreactivity indicating colocalization of Ang-1 and Ang-2. D, At 6 hours, cytoplasmic colocalization of Ang-1 and Ang-2 (yellow) is also present in polymorphonuclear leukocytes around a pial vein, which shows endothelial Ang-1 and minimal Ang-2 in the red channel $(E)$. At Day 4 , perivascular macrophages ( $F$ ) show cytoplasmic colocalization of Ang-1 and Ang-2 (yellow). The vessel endothelium shows focal Ang-1 immunoreactivity. The corresponding red channel show Ang-2 localization in these macrophages (F, inset). G, A Day 4 lesion showing colocalization of Ang-1 and Ang-2 in perivascular glial processes (arrowheads), which surround microvessels $(v)$ and the large vessel $(v)$ present on the left-hand edge of the panel. $H, A$ Day 4 lesion showing colocalization of Ang-1 and Ang-2 in pial vessels. A Day 2 lesion (I) showing a vessel with increased permeability to fibronectin (red) with extravasation of this protein into the surrounding brain. Note absence of endothelial Ang-1 while polymorphonuclear leukocytes in the surrounding brain show cytoplasmic Ang-1 (green). J, A Day 4 lesion showing fibronectin extravasation from a vessel, which lacks endothelial Ang-2, while the surrounding cells that are both endothelial cells and macrophages show cytoplasmic Ang-2 (green). K, Pial vessels of a Day 4 lesion show endothelial colocalization of Ang-2 and fibronectin (yellow). The antifibronectin antibody demonstrates both plasma and cellular fibronectin; therefore, localization of fibronectin to the vessel wall indicates that these vessels do not show blood-brain barrier breakdown to fibronectin. Scale bar: $\mathrm{A}$ to $\mathrm{K}=50 \mu \mathrm{m}$. 
regulate the function of these cell types in steady states by an autocrine mechanism. To our knowledge, this is the first report of angiopoietin localization in the choroid plexuses and the ependyma.

\section{Ang-1 and Ang-2 Expression During Cerebral Angiogenesis}

Ang-1 and Ang-2 expression within the lesion was inversely related during the 6-day period after injury. The relative decrease in Ang-1 compared with Ang-2 mRNA at 6 hours after injury may have a role in the initial steps of vascular remodeling and angiogenesis, namely breakdown of the preexisting endothelial junctions. Also, because Ang-1 promotes endothelial cell survival (Hayes et al, 1999; Kwak et al, 1999; Papapetropoulos et al, 2000), and Ang-2 has been observed just before the onset of vascular apoptosis (Cohen et al, 2001; Zagzag et al, 2000), the significant decrease in Ang-1 relative to Ang-2 mRNA at 6 hours after injury compared with normal levels may induce regression of the intrinsic lesion vessels.

The abrupt up-regulation of Ang-2 mRNA during non-neoplastic cerebral angiogenesis has been described previously in human arteriovenous malformations (Hashimoto et al, 2001), after systemic hypoxia in rat brain (Mandriota et al, 2000), and in infarcts produced by middle cerebral artery occlusion in the rat (Beck et al, 2000). Unlike the present study, Ang-1 mRNA levels in these models were similar or only slightly increased compared with control brains. This difference could be a result of the particular phase after injury when Ang-1 mRNA was assessed. Although Ang-2 protein localization was mild in vessels at Day 2, the marked Ang-2 immunoreactivity in inflammatory cells could account for the early surge in Ang-2 mRNA observed using RT-PCR. The progressive relative increase in Ang-1 compared with Ang-2 mRNA and protein from 2 to 6 days after injury may play a role in later stages of angiogenesis and vascular remodeling after cortical cold injury, namely maturation of the newly formed vessels by association of cerebral endothelium with periendothelial cells such as pericytes and astrocytes.

Vascular endothelial growth factor-A (VEGF-A) expression at the mRNA and protein level also increases after a cortical cold injury (Nag et al, 1997, 2002). Angiopoietins and VEGF are known to interact during pathologic angiogenesis (Peters, 1998), and they are both indispensable for this process (Siemeister et al, 1999). Furthermore, concurrent up-regulation of angiopoietins and VEGF occurs during both neoplastic (Ding et al, 2001) and non-neoplastic (Mandriota et al, 2000) cerebral angiogenesis. Therefore, the concurrent increase in VEGF and angiopoietins facilitates cerebrovascular angiogenesis in this model as well.

\section{Ang-1 and Ang-2 Protein in Inflammatory Cells}

This is the first report of cytoplasmic localization of Ang-1 and Ang-2 proteins in PMNs and macrophages after brain injury. This cytoplasmic localization is not a result of the high myeloperoxidase content of leukocytes because cytoplasmic Ang-1 and Ang-2 and colocalization of these proteins in inflammatory cells was observed by immunofluorescence as well. Ang-1 and Ang-2 immunoreactivity was previously reported outside the brain in macrophage-like mesenchymal cells during the angiogenesis occurring in human pyogenic granuloma (Yuan et al, 2000).

Inflammatory cells are known to play a key role in angiogenesis after injury. Activated macrophages are known to influence each phase of the angiogenic process, by producing alterations of the local extracellular matrix and by inducing endothelial cell migration or proliferation and inhibition of vascular growth leading to the formation of differentiated capillaries (Polverini et al, 1977; Sunderkotter et al, 1994). Our previous studies have shown increased expression of VEGF-A and -B in inflammatory cells in the same time frame as the present study (Nag et al, 1997, 2002). Hence a beneficial effect of inflammatory cells after injury is that they are a source of multiple angiogenic growth factors. Because angiopoietins are known to alter phosphorylation of endothelial junctional proteins of noncerebral endothelium (Gamble et al, 2000), expression of angiopoietins by macrophages may be involved in tight junctional remodeling after cold injury. Macrophage-mediated tight junctional modulation is critical in the process of diapedesis (Edens and Parkos, 2000), and this process may be partly mediated by Ang-2. This hypothesis is supported by the observation that Ang- 1 inhibits TNF- $\alpha$-stimulated leukocyte transmigration, and thus acts as an anti-inflammatory agent in noncerebral endothelial cells (Gamble et al, 2000).

Both Ang-1 and Ang-2 protein were also localized in peri-lesion astrocytes at Day 4. Although Ang-1 mRNA has been localized in neoplastic astrocytes (Stratmann et al, 1998; Zagzag et al, 1999) and Ang-2 mRNA in peri-infarct astrocytes by in situ hybridization, there are no previous reports of localization of Ang-1 or 2 proteins in nonneoplastic astrocytes.

\section{Endothelial Ang-1 and Ang-2 during BBB Breakdown}

The time course of BBB breakdown was similar to previous observations, with fibronectin extravasation being present at 6 hours and Days 2 to 4 after injury and decreasing thereafter ( $\mathrm{Nag}$ et al, 1997, 2001, 2002). Although Ang-1 mRNA was not significantly altered at 6 hours and Day 2 after injury, loss of endothelial Ang-1 protein was observed as early as 6 hours and the number of Ang-1 immunoreactive vessels decreased from $99 \%$ in controls to $52 \%$ at Day 2. The time course of this reduction coincided with maximum BBB breakdown. Although decreased Ang-1 mRNA has been associated with BBB breakdown (Zhang et al, 2002b), this study provides definitive evidence that vessels demonstrating BBB breakdown show loss of or reduced endothelial Ang-1 and Tie-2 proteins. The importance of Ang-1 in maintaining the structural and permeability properties of normal vessels has been discussed previously. Therefore, the 
relative decrease in Ang-1 to Ang-2 mRNA and Ang-1 protein in the early phase after cold injury contributes to disruption of endothelial tight junctions and dissociation of periendothelial supporting cells from endothelium, leading to vascular destabilization and BBB breakdown. This phase of BBB breakdown promotes angiogenesis and repair because the extravasated proteins form a matrix, which facilitates endothelial migration. Although vessels leaking protein did not show endothelial Ang-2, there was an increase in both Ang-2 mRNA and protein at the lesion site at 6 hours and Day 2, suggesting that Ang-2 may be involved in BBB breakdown; however, further studies are required to prove this hypothesis.

VEGF-A has also been implicated in the BBB breakdown occurring after cortical cold injury (Nag et al, $1997,2002)$. One of the mechanisms by which VEGF induces vascular permeability is targeting of endothelial junctional proteins (Antonetti et al, 1999; Kevil et al, 1998). Because Ang-1 modulates phosphorylation of endothelial junctional proteins (Gamble et al, 2000), a decrease in Ang-1 or an increase in Ang-2 in the early phase after injury may allow for the permeabilityinducing actions of VEGF. This hypothesis is supported by the observation that Ang-1 overexpression inhibits the permeability-inducing effect of VEGF (Thurston et al, 1999). Also, mouse cornea neovascularization assays have shown that VEGF-induced neovasculature is not associated with periendothelial cells, whereas abundant periendothelial cells are observed in neovasculature that is coinduced with both Ang-1 and VEGF (Asahara et al, 1998).

The relative increase in Ang-1 mRNA and protein compared with Ang-2 at 4 and 6 days after injury coincides with BBB restoration. Assuming that Ang-1 has a similar function in cerebral vessels as in peripheral vessels, the increase in Ang-1 in later phases after injury may promote association of newly formed vessels with periendothelial pericytes and astrocytes, and therefore lead to restoration of the BBB. Furthermore, because Ang-2 activates Tie-2 at high concentrations (Kim et al, 2000) and after prolonged exposure (Teichert-Kuliszewska et al, 2001), Ang-2 in later phases after injury may have an agonistic effect on Tie-2 and hence may promote BBB restoration.

In conclusion this study demonstrates altered expression of Ang-1 and Ang-2 during BBB breakdown and cerebral angiogenesis and demonstrates that Ang-1 is an important factor in maintaining normal homeostasis in the brain. Lack of Ang-1 and increased VEGF are important factors in the pathogenesis of BBB breakdown and subsequent vasogenic edema formation after brain injury. Thus Ang- 1 therapy may have therapeutic potential in reducing BBB breakdown and the ensuing edema after massive brain injury. In this context it is interesting that a recent study reports reduction in BBB leakage to Evans blue and a reduction in infarct volume in mice injected with a recombinant adenovirus expressing Ang- 1 before middle cerebral artery (MCA) occlusion (Zhang et al, 2002a).

\section{Materials and Methods}

\section{Cortical Cold Injury}

Cold injury was produced in male Wistar rats (180 to $200 \mathrm{gm}$ ) as described previously (Nag et al, 2001). Rats were anesthetized by inhalation of methoxyflurane (Metophane; Janssen Pharmaceutica, Titusville, New Jersey). A craniotomy was performed in the left parietal bone midway between the lambdoid and coronal sutures using a dental drill with a 2.3-mm bit (Horico, Berlin, Germany). The cold probe, consisting of a $20-\mathrm{ml}$ syringe with a blunt copper wire tip, was filled with liquid nitrogen and applied to the craniotomy site for 45 seconds. The incision was sutured, and buprenorphine hydrochloride (Buprenex, $0.033 \mathrm{ml}$ per 100 gm body weight; Reckitt \& Colman Pharmaceuticals Inc., Hull, England) was injected ip for analgesia. This protocol was in accordance with the guidelines set by the Canadian Council on Animal Care and was further approved by the local animal care committee.

\section{RT-PCR}

Rats were decapitated in groups of five, together with five controls, at 6 hours and at 2, 4, and 6 days after injury. Cortical samples weighing about $20 \mathrm{mg}$ were obtained from the lesion and peri-lesion areas and the corresponding area of the contralateral hemisphere. Samples were flash-frozen in liquid nitrogen and stored at $-80^{\circ} \mathrm{C}$.

RNA extraction was performed using the RNeasy Kit (QIAGEN, Mississauga, Ontario, Canada) according to the provided protocol. Absorbance of RNA was measured at 260 and $280 \mathrm{~nm}$ using a SpectraMax-250 spectrophotometer and the SOFTmax-Pro computer software (Global Medical Instrumentation Inc., Clearwater, Minnesota). RNA concentration and purity were estimated as follows: $[\mathrm{RNA}]=\mathrm{OD}_{260} \times$ (dilution factor) $\times 40$, purity $=\mathrm{OD}_{260} / \mathrm{OD}_{280}$.

Reverse-transcription was performed in $40 \mu \mathrm{l}$ of reaction volume containing $2 \mu \mathrm{g}$ of sample RNA, 0.450 $\mu \mathrm{g}$ random primers (GibcoBRL, Burlington, Ontario, Canada), $200 \mu \mathrm{M}$ deoxynucleotide triphosphate mixture, $400 \mathrm{U}$ of Moloney murine leukemia virus reverse transcriptase (GibcoBRL), $1 \mathrm{~mm}$ dithiothreitol, and $22640 \mathrm{U}$ of RNAguard RNase inhibitor (Amersham Pharmacia Biotechnology, Piscataway, New Jersey).

PCR amplification was performed in $50 \mu \mathrm{l}$ total volume, containing 2 to $10 \mu \mathrm{l}$ of reverse transcription product, $200 \mu \mathrm{M}$ deoxynucleotide triphosphate mixture, 50 pmol of each primer (Amersham Pharmacia Biotechnology), and $2.5 \mathrm{U}$ of Taq polymerase (Amersham Pharmacia Biotechnology). The sequence of primers was as follows: Ang-1, sense 5'-CACGACAGACCAGTAC AACACAAACG-3' and antisense 5'-GACGACTGTTG TTGGTGGTAGCTCT-3'; Ang-2, partially degenerate primers, sense $5^{\prime}-G T(G T) G A(C T) T T(C T) C A G A G(A C G T)$ AC(ACGT)TGG-3' and antisense 5'-CGA(AG)TAG CC(GT)GA(ACGT)CC(CT)TTCCA-3'; Tie-2, sense 5'CAGGACCTTCACAACAGCTTCTATCGGACT-3' and antisense 5'-CTGTCGAAGAATGTCACTAAGGGTCC- 
AAGC-3'; and glyceraldehyde-3-phosphate dehydrogenase (GAPDH), sense 5'-CTCTAAGGCTGTGGCA AGGTCAT-3' and antisense 5'-GAGATCCACCAC CCTGTTGCTGTA-3'. Amplification cycles were 30 for Ang-1, Ang-2, and Tie-2, and 25 for GAPDH. The sizes of the PCR products were $570 \mathrm{bp}, 450 \mathrm{bp}, 322 \mathrm{bp}$, and 343 bp for Ang-1, Ang-2, Tie-2, and GAPDH, respectively. The amplified cDNA segments were run on $2 \%$ agarose gels containing $4 \%$ ethidium bromide. Signals were visualized using a gel scanning system (Bio-Rad Laboratories, Hercules, California).

\section{Immunohistochemistry}

Rats were killed in groups of five, together with five controls, at 6 hours and at 2,3,4, and 6 days after injury. Rats were anesthetized with Metophane and perfused with $3 \%$ paraformaldehyde in $0.1 \mathrm{M}$ phosphate buffer $(\mathrm{pH} 7.3)$ at a pressure of $110 \mathrm{mmHg}$, by a cannula in the ascending aorta. A coronal slab of brain containing the cold-injury site was processed for paraffin sectioning using standard techniques. Sections (6 $\mu \mathrm{m})$ were stained with hematoxylin and eosin for histologic analysis, and adjacent sections were used for immunohistochemistry.

The indirect streptavidin-biotin peroxidase method was used, and paraffin sections were pretreated with $0.5 \%$ pepsin in $0.01 \mathrm{M} \mathrm{HCl}$ for 30 minutes at $37^{\circ} \mathrm{C}$ before overnight incubation in primary antibody at $4^{\circ} \mathrm{C}$. Dilutions of the antibodies used were as follows: polyclonal goat Ang-1 and Ang-2 (Santa Cruz Biotechnology, Santa Cruz, California), 1:100; polyclonal rabbit Tie-2 (Santa Cruz Biotechnology), 1:575; and fibronectin (Gibco BRL), 1:400. Anti-Ang-2 antibodies directed against both the carboxy-terminus (C-19) and the amino terminus ( $\mathrm{F}-18)$ of the Ang-2 protein were used. Diaminobenzidine was used as a substrate for these reactions.

Dual labeling was also performed in brains of control rats and cold-injured rats at 6 hours and at 2 and 4 days after injury for (1) Ang-1 and Ang-2, (2) Ang-1 and fibronectin, and (3) Ang-2 and fibronectin. Details of the dual method have been described previously (Nag et al, 2002). Stretpavidin-Alexa Fluor 488 (Molecular Probes Inc., Eugene, Oregon) and goat antirabbit-Cy 3 (Jackson Immunoresearch Laboratories Inc., West Grove, Pennsylvania) were used as link antibodies and emitted a green and red fluorescence, respectively. Sections were analyzed using a Nikon Optiphot microscope and a MRC 600 confocal laserscanning microscope (Bio-Rad Laboratories Canada Ltd., Mississauga, Canada). Images were merged using Adobe Photoshop 7.0 software.

Negative controls included omission of primary antibody, neutralization of the primary antibody with blocking peptides, and use of nonimmune serum. Positive controls used were the lung for Ang-1, the prostate for Ang-2, and the ovary for both Ang-1 and Ang-2.

\section{Densitometry}

An MCID Image Analyzer 5+ (Imaging Research Inc., St. Catharines, Ontario, Canada) was used. Density was estimated as [optical density] $\times$ [area] for each time period after injury. To compare density of signals on the same gel, all values were normalized to their corresponding GAPDH signals. To compare RT-PCR results on two different gels, the signal density of 15 normal samples was averaged and assigned the arbitrary value of 1 . All other signals were calculated as ratio of normal density.

\section{Quantitative Morphometry}

The total number of cross-sectional profiles of vessels, macrophages, and PMNs within the lesion and those containing Ang-1 and Ang-2 were counted at Days 2 and 4. Counts were done three times in three high-power fields of $0.283 \mathrm{~mm}^{2}$ using a Leitz Orthoplan microscope (Wetzlar, Germany) fitted with a $\times 40$ objective. Results were expressed as the percentage of immunostained vessels, PMNs, and macrophages.

\section{Statistical Analysis}

ANOVA with Tukey's post-hoc test (SYSTAT 9; SPSS Science Inc., Chicago, Illinois) was used to determine the significance between the means of control and test groups. Differences were considered significant at a value of $p<0.05$. Pearson's correlation test was used to correlate changes in Ang-1 and Ang-2 mRNA expression during 6 hours to 6 days after injury.

\section{References}

Antonetti DA, Barber AJ, Hollinger LA, Wolpert EB, and Gardner TW (1999). Vascular endothelial growth factor induces rapid phosphorylation of tight junction proteins occludin and zonula occluden 1: A potential mechanism for vascular permeability in diabetic retinopathy and tumors. J Biol Chem 274(33):23463-23467.

Asahara T, Chen D, Takahashi T, Fujikawa K, Kearney M, Magner M, Yancopoulos GD, and Isner JM (1998). Tie2 receptor ligands, angiopoietin-1 and angiopoietin-2, modulate VEGF-induced postnatal neovascularization. Circ Res 83:233-240.

Audero E, Cascone I, Zanon I, Previtali SC, Piva R, Schiffer D, and Bussolino $F$ (2001). Expression of angiopoietin-1 in human glioblastomas regulates tumor-induced angiogenesis. Thromb Vasc Biol 21:536-541.

Beck $\mathrm{H}$, Acker T, Wiessner C, Allergrini PR, and Plate $\mathrm{K}$ (2000). Expression of angiopoietin-1, angiopoietin-2, and Tie receptors after middle cerebral artery occlusion in the rat. Am J Pathol 157:1473-1483.

Cheung AH, Stewart RJ, and Marsden PA (1998). Endothelial Tie2/Tek ligands angiopoietin-1 (ANGPT1) and angiopoietin-2 (ANGPT2): Regional localization of the human genes to 8q22.3-q23 and 8p23. Genomics 48:389-391.

Cohen B, Barkan D, Levy Y, Goldberg I, Fridman E, Kopolovic J, and Rubinstein M (2001). Leptin induces angiopoietin-2 expression in adipose tissues. J Biol Chem 276(11):7697-7700. 
Davis S, Aldrich TH, Jones PF, Acheson A, Compton DL, Jain V, Ryan TE, Bruno J, Radziejewski C, Maisonpierre PC, and Yancopoulos GD (1996). Isolation of angiopoietin-1, a ligand for the Tie2 receptor, by secretion-trap expression cloning. Cell 87:1161-1169.

Ding $\mathrm{H}$, Roncari L, Wu X, Lau N, Shannon P, Nagy A, and Guha A (2001). Expression and hypoxic regulation of angiopoietins in human astrocytomas. Neuro-Oncol 3(1):1-10.

Edens HA and Parkos CA (2000). Modulation of epithelial and endothelial paracellular permeability by leukocytes. Adv Drug Deliv Rev 41:315-328.

Gamble JR, Drew J, Trezise L, Underwood A, Parsons M, Kasminkas L, Rudge L, and Yancopoulos G, and Vadas MA (2000). Angiopoietin-1 is an antipermeability and antiinflammatory agent in vitro and targets cell junctions. Circ Res 87(7):603-607.

Goede V, Schmidt T, Kimmina S, Kozian D, and Augustin HG (1998). Analysis of blood vessel maturation processes during cyclic ovarian angiogenesis. Lab Invest 78:1385-1394.

Grosios K, Leek JP, Markham AF, Yancopoulos GD, and Jones PF (1999). Assignment of ANGPT4, ANGPT1, and ANGPT2 encoding angiopoietins 4,1 and 2 to human chromosome bands 20p13, 8q22.3 $\rightarrow$ q23 and 8p23.1, respectively, by in situ hybridization and radiation hybrid mapping. Cytogenet Cell Genet 84:118-120.

Hashimoto T, Lam T, Boudreau NJ, Bollen AW, Lawton MT, and Young WL (2001). Abnormal balance in the angiopoietintie2 system in human brain arteriovenous malformations. Circ Res 89(2):111-113.

Hayes AJ, Huang WQ, Mallah J, Yang D, Lippman ME, and Li LY (1999). Angiopoietin-1 and its receptor Tie-2 participate in the regulation of capillary-like tubule formation and survival of endothelial cells. Microvasc Res 58:224-237.

Kevil CG, Payne DK, Mire E, and Alexander JS (1998). Vascular permeability factor/vascular endothelial growth factor-mediated permeability occurs through disorganization of endothelial junctional proteins. J Biol Chem 273(24): 15099-15103.

Kim I, Kim JH, Moon SO, Kwak HJ, Kim NG, and Koh GY (2000). Angiopoietin-2 at high concentration can enhance endothelial cell survival through the phosphatidylinositol 3'kinase/Akt signal transduction pathway. Oncogene 19(39): 4549-4552.

Kwak HJ, So JN, Lee SJ, Kim I, and Koh GY (1999). Angiopoietin-1 is an apoptosis survival factor for endothelial cells. FEBS Lett 448:249-253.

Maisonpierre PC, Suri C, Jones PF, Bartunkova S, Wiegand SJ, Radziejewski C, Compton D, McClain J, Aldrich TH, Papadopoulos N, Daly TJ, Davis S, Sato TN, and Yancopoulos GD (1997). Angiopoietin-2, a natural antagonist for Tie2 that disrupts in vivo angiogenesis. Science 277:55-60.

Mandriota SJ, Pyke C, Di Sanza C, Quinodoz P, Pittet B, and Pepper MS (2000). Hypoxia-inducible angiopoietin-2 expression is mimicked by iodonium compounds and occurs in the rat brain and skin in response to systemic hypoxia and tissue ischemia. Am J Pathol 156:2077-2089.

Nag S (1996). Cold-injury of the cerebral cortex: Immunolocalization of cellular proteins and blood-brain barrier permeability studies. J Neuropath Exp Neurol 55(8):880-888.
Nag S (2002). The blood-brain barrier and cerebral angiogenesis: Lessons from the cold-injury model. Trends Mol Med 8:38-44.

Nag S, Eskandarian MR, Davis J, and Eubanks JH (2002). Differential expression of vascular endothelial growth factor-A (VEGF-A) and VEGF-B after brain injury. J Neuropathol Exp Neurol 61:778-788.

Nag S, Picard P, and Stewart DJ (2001). Expression of nitric oxide synthases and nitrotyrosine during blood-brain barrier breakdown and repair after cold-injury. Lab Invest 81(1):4149.

Nag S, Takahashi JL, and Kilty DW (1997). Role of vascular endothelial growth factor in blood-brain barrier breakdown and angiogenesis in brain trauma. J Neuropathol Exp Neurol 56(8):912-921.

Papapetropoulos A, Fulton D, Mahboubi K, Kalb RG, O'Connor DS, Li F, Altieri DC, and Sessa WC (2000). Angiopoietin-1 inhibits endothelial cell apoptosis via the Akt/survivin pathway. J Biol Chem 275:9102-9105.

Patan S (1998). TIE1 and TIE2 receptor tyrosine kinases inversely regulate embryonic angiogenesis by the mechanism of intussusceptive microvascular growth. Microvasc Res 56:1-21.

Peters KG (1998). Vascular endothelial growth factor and the angiopoietins: Working together to build a better blood vessel. Circ Res 83:342-343.

Polverini PJ, Cotran RS, Gimbrone MA Jr, and Unanue ER (1977). Activated macrophages induce vascular proliferation. Nature (Lond) 269:804-806.

Siemeister G, Schirner M, Weindel K, Reusch P, Menrad A, Marme D, and Martiny-Baron G (1999). Two independent mechanisms essential for tumor angiogenesis: Inhibition of human melanoma xenograft growth by interfering with either the vascular endothelial growth factor receptor pathway or the Tie-2 pathway. Cancer Res 59:3185-3191.

Stratmann A, Risau W, and Plate KH (1998). Cell typespecific expression of angiopoietin-1 and angiopoietin-2 suggests a role in glioblastoma angiogenesis. Am J Pathol 153:1459-1466.

Sunderkotter C, Steinbrink K, Goebeler M, Bhardwaj R, and Sorg C (1994). Macrophages and angiogenesis. J Leukoc Biol 55:410-422.

Suri C, Jones PF, Patan S, Bartunkova S, Maisonpierre PC, Davis S, Sato TN, and Yancopoulos GD (1996). Requisite role of angiopoietin-1, a ligand for the TIE2 receptor, during embryonic angiogenesis. Cell 87:1171-1180.

Suri C, McClain J, Thurston G, McDonald DM, Zhou H, Oldmixon EH, Sato TN, and Yancopoulos GD (1998). Increased vascularization in mice overexpressing angiopoietin-1. Science 282:468-471.

Teichert-Kuliszewska K, Maisonpierre PC, Jones N, Campbell AIM, Master Z, Bendeck MP, Alitalo K, Dumont DJ, Yancopoulos GD, and Stewart DJ (2001). Biological action of angiopoietin-2 in a fibrin matrix model of angiogenesis is associated with activation of Tie-2. Cardiovasc Res 49:659670.

Thurston G (2002). Complementary actions of VEGF anf angiopoietin-1 on blood vessel growth and leakage. J Anat 200:575-580. 
Thurston G, Suri C, Smith K, McClain J, Sato TN, Yancopoulos GD, and McDonald DM (1999). Leakage-resistant blood vessels in mice transgenically overexpressing angiopoietin-1. Science 286:2511-2514.

Wong AL, Haroon ZA, Werner S, Dewhirst MW, Greenberg CS, and Peters KG (1997). Tie2 expression and phosphorylation in angiogenic and quiescent adult tissues. Circ Res 81:567-574.

Yuan K, Jin YT, and Lin MT (2000). Expression of Tie-2, angiopoietin-1, angiopoietin-2, ephrinB2 and EphB4 in pyogenic granuloma of human gingiva implicates their roles in inflammatory angiogenesis. J Periodontal Res 35(3):165-171.

Zagzag D, Amirnovin R, Greco MA, Yee H, Holash J, Wiegand SJ, Zabski S, Yancopoulos GD, and Grumet M (2000). Vascular apoptosis and involution in gliomas precede neovascularization: A novel concept for glioma growth and angiogenesis. Lab Invest 80:837-849.
Zagzag D, Hooper A, Friedlander DR, Chan W, Holash J, Wiegand SJ, Yancopoulos GD, and Grumet M (1999). In situ expression of angiopoietins in astrocytomas identifies angiopoietin-2 as an early marker of tumor angiogenesis. Exp Neurol 159:391-400.

Zhang ZG, Zhang L, Croll SD, and Chopp M (2002a). Angiopoietin-1 reduces cerebral blood vessel leakage and ischemic lesion volume after focal cerebral embolic ischemia in mice. Neuroscience 113:683-687.

Zhang ZG, Zhang L, Tsang W, Soltanian-Zadeh H, Morris D, Zhang R, Goussev A, Powers C, Yeich T, and Chopp M (2002b). Correlation of VEGF and angiopoietin expression with disruption of blood-brain barrier and angiogenesis after focal cerebral ischemia. J Cereb Blood Flow Metab 22:379_ 392. 